\title{
Relación entre capacitación y actitud hacia los riesgos laborales en el sector construcción del área metropolitana de Bucaramanga
}

Relationship between training and attitude towards occupational risks in the building industry in the metropolitan area of Bucaramanga
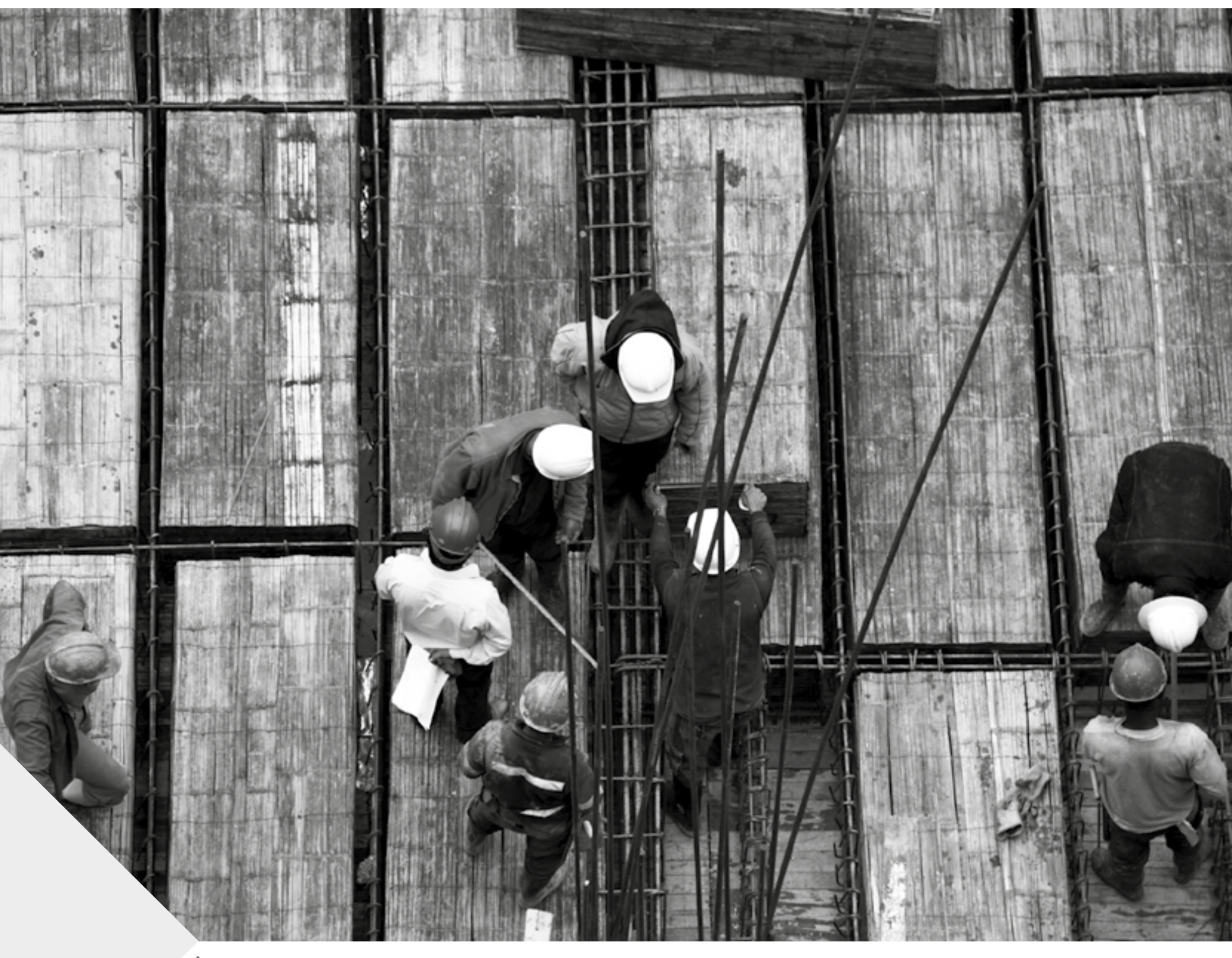


\title{
Relación entre capacitación y actitud hacia los riesgos laborales en el sector construcción del área metropolitana de Bucaramanga ${ }^{1}$ Relationship between training and attitude towards occupational risks
in the building industry in the metropolitan area of Bucaramanga
}

\author{
Germán M. Argüello-López², Johanna M. Uribe-Bermúdez³, \\ Manuel Valdivieso-Guerrero ${ }^{4}$
}

'Corporación Universitaria de Investigación y Desarrollo, Bucaramanga, Colombia

Artículo recibido en junio de 2016; artículo aceptado en septiembre de 2016

Citación del artículo: Argüello López, G; Uribe Bermúdez,J. \&Valdivieso Guerrero, M. (2017). Relación entre capacitación y actitud hacia los riesgos laborales en el sector construcción del área metropolitana de Bucaramanga: Un estudio de caso. I+D Revista de Investigaciones, 9(1), 14 - 26

\section{Resumen}

Numerosas investigaciones han demostrado la relación entre actitudes, comportamientos y accidentes laborales; este estudio fue desarrollado con el fin de determinar la relación entre capacitación y actitud hacia los riesgos laborales en pymes del sector construcción del área metropolitana de Bucaramanga, Colombia. Este estudio tuvo un enfoque cuantitativo, no experimental, de corte transversal y de alcance correlacional. Fue diseñada una escala de veinte ítems para medir la actitud hacia los riesgos laborales de trabajadores del sector construcción. Este documento de trabajo describe los resultados de la aplicación de la escala en ciento trece trabajadores de tres pymes del sector construcción de Bucaramanga. Los resultados probaron la relación entre capacitación y actitud hacia los riesgos laborales; además, permitieron comprobar la fiabilidad de la escala diseñada.

Palabras clave: Actitud, riesgos laborales, pymes, construcción.

\footnotetext{
Abstract

Numerous studies have shown the relationship between attitudes, behaviors and occupational accidents. This study was developed in order to determine the relation between training and attitude towards occupational risks in SMEs of the building industry in the metropolitan area of Bucaramanga. This study was a quantitative approach, not experimental, of a cross-sectional and correlational scope. It was designed a 20-item scale to measure attitude toward occupational hazards of workers in the building industry. This working paper describes the results of the application of the scale in 113 workers from 3 SMEs in the building industry Bucaramanga. The results showed the 1. Artículo de investigación resultado de un proyecto de investigación del grupo de investigación Sinergia, financiado por la Corporación Universitaria de Investigación y Desarrollo - UDI, Bucaramanga, Colombia. Dirección: Calle 9 n. ${ }^{\circ}$ 23-55, PBX: 6352525. Fecha de inicio: agosto de 2015, fecha de terminación: junio de 2016.

2. Ingeniero industrial, UIS. Magister en Entornos Virtuales de Aprendizaje, Universidad de Panamá. Docente- investigador del grupo Sinergia. Corporación Universitaria de Investigación y Desarrollo, Bucaramanga, Colombia. Dirección: calle 9 n. ${ }^{\circ}$ 23-55, PBX: 6352525. Correo electrónico institucional: garguello1@udi.edu.co.

3. Ingeniero industrial, UPB Seccional Bucaramanga. Docente- investigadora del grupo Sinergia. Corporación Universitaria de Investigación y Desarrollo, Bucaramanga, Colombia. Dirección: calle 9 n. ${ }^{\circ} 23-55$, PBX: 6352525. Correo institucional: juribe1@udi.edu.co.

4. Ingeniero industrial, UDI. Joven-investigador del grupo Sinergia. Corporación Universitaria de Investigación y Desarrollo, Bucaramanga, Colombia. Dirección: calle 9 n.²3-55, PBX: 6352525. Correo institucional: mvaldivieso1@udi.edu.co.
} 
relationship between attitude towards training and occupational hazards; also it allowed to check the reliability of the scale designed.

Keywords: Attitude, occupational hazards, SMEs, construction.

El sector construcción incluye la construcción de edificios, las obras de ingeniería civil así como las actividades especializadas para la construcción de edificios y obras de ingeniería civil. Según reporte de la Cámara de Comercio de Bucaramanga, este sector económico del departamento de Santander se compone de 39 empresas grandes, 166 medianas, 522 pequeñas y 3354 microempresas (Cámara de Comercio de Bucaramanga, 2016). En el 2015 había en el área metropolitana de Bucaramanga 3463 empresas del sector construcción afiliadas al Sistema General de Riesgos Laborales - SGRL, mediante las cuales se encontraban afiliados 47118 empleados. Como es de esperar en un sector tan intensivo en mano de obra, las cifras de accidentalidad laboral y de enfermedades laborales del área metropolitana de Bucaramanga fueron altas: 6517 accidentes laborales, 22 enfermedades laborales y 7 muertes calificadas (Fasecolda, 2016).

Un indicador que puede dar mayor claridad sobre la situación de accidentalidad del sector construcción del área metropolitana de Bucaramanga con respecto al nivel nacional y a todos los sectores en el nivel local y nacional, es el promedio anual de accidentes por empresa. La Figura 1 muestra que el promedio anual de accidentes por empresa del sector construcción del área metropolitana de Bucaramanga ha venido disminuyendo, pero se ha mantenido por encima del promedio anual del sector construcción del país y por encima del promedio anual para todos los sectores, tanto del área metropolitana como a nivel nacional.

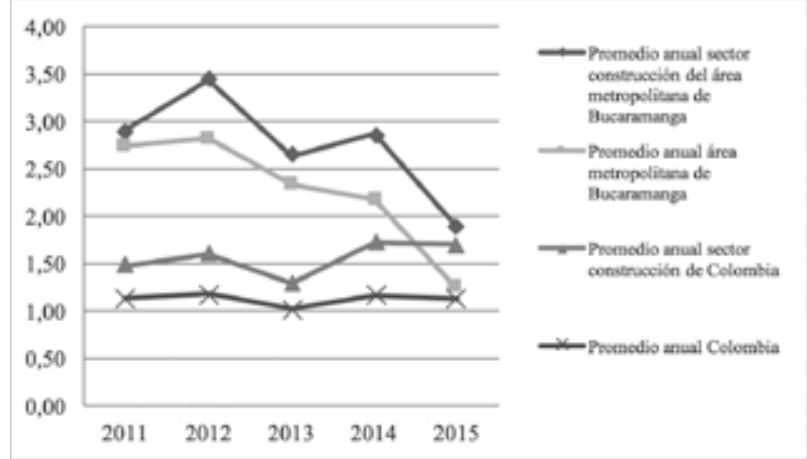

Figura 1.

Promedio anual de accidentes por empresa área metropolitana de Bucaramanga -AMB y nacional para el sector construcción y para todos los sectores.

Fuente: Elaboración propia con base en datos de Fasecolda.
Pucci (2007) propone una clasificación de las investigaciones sobre seguridad en el trabajo en tres ejes fundamentales: el individuo, el puesto de trabajo y la organización. Pucci explica que las investigaciones respecto de los individuos han girado en torno al análisis de la fiabilidad de los comportamientos partiendo de cuatro determinantes: la personalidad, la motivación, el aprendizaje y la decisión. Puya (2001) confirma esto afirmando que en la prevención de riesgos laborales intervienen importantes aspectos psicosociales tales como las actitudes, los hábitos, la percepción del riesgo y la percepción de control de este, y el nivel de satisfacción de los empleados, entre otros.

Siguiendo esta línea, Wilde (1982) propuso la teoría del "equilibrio de riesgo" la cual estima que los individuos perciben sus comportamientos como más o menos riesgosos, y los adaptan para que se correspondan a un nivel de riesgo deseado. También por esta línea surge la "ilusión de la invulnerabilidad", que explica por qué la mayoría de las personas creen que tienen menos posibilidades que sus pares de que les ocurran acontecimientos negativos (Sánchez, Rubio, Páez \& Blanco, 1998). Sánchez et al. (1998) concluyen que este "optimismo ilusorio" reduce la probabilidad de que el individuo ejecute las conductas adecuadas para reducir el riesgo real que sobreviene al verse implicado en determinadas situaciones. En consecuencia, con esta teoría Weinstein (1989) plantea la prevención de riesgos laborales como un proceso de "aprendizaje individual" en tres fases: toma de conciencia individual de los riesgos, conocimiento general de las consecuencias de un accidente y conocimiento específico de las consecuencias que un accidente puede tener en sí mismo, de manera que cuanto más avanza el individuo en estas tres fases de "aprendizaje individual", más tendencia tendrá a desarrollar un comportamiento que tenga en cuenta los riesgos reales de su actividad.

Las actitudes hacen parte de lo que se denomina "cultura de seguridad en el trabajo", definida como el producto de los valores, actitudes, percepciones, competencias y comportamientos individuales y de grupo que pueden determinar el compromiso y el estilo y el dominio del sistema de gestión de la salud y la seguridad de una organización (Health and Safety Commission, 1993).

Vallerand (1994) define la actitud en términos de los siguientes elementos: a) es un constructo o variable no 
observable directamente; b) implica una relación entre aspectos cognitivos, afectivos y conativos; c) motiva y orienta a la acción y también influye en la percepción y el pensamiento; d) es aprendida; e) es perdurable; $y$, f) tiene un componente de evaluación o afectividad simple de agrado-desagrado. Bentler y Speckart (1981) postularon que la actitud influye en el comportamiento tanto directamente como a través de la intención de actuar.

Se han desarrollado numerosos estudios para determinar la relación entre actitudes y accidentes laborales en profesionales de la salud (Alcántara, Rodríguez,González, \& Clapes, 2013; Cortijo, Gómez \& Samalvides, 2010; De La Torre \& Tarabla, 2015; Franco, Marín, Ocampo, Quiroz \& Díaz, 2007; López, Andrade, Tarabla, Signorini \& Molineri, 2014; Olivos, Ávila \& Arana, 2008), industria manufacturera (Soto \& Mogollón, 2005; Taufek, Zulkifle \& Kadir, 2016; Zamorano, Parra, Peña, \& Castillo, 2009), sector construcción (Corrales, Sánchez, \& Toledo, 2014; Ismail, F., Ahmad, Janipha \& Ismail, R. 2012; Martínez,\& Montero, 2015; Meza\& Camargo, 2015; Shin, Lee, Park, Moon \& Han, 2014; Zahoor, Chan, Utama \& Gao, 2015; Zin \& Ismail, 2012) y otros sectores (Burgos, 2014; Du \& Sun, 2012; Fernández,Mayayo, Chamarro \& Virgili 2015; Molina, 2012; Ospina, Manrique, \& Ariza, 2009).

Uno de los medios para mejorar la actitud de los trabajadores acerca de la prevención de los riesgos laborales es la capacitación, ya que esta proporciona al trabajador conocimientos específicos que le ayudan a prevenir los accidentes, llevándolo a la concientización y al logro de comportamiento seguros (Chen \& Tian, 2012). En línea con estos planteamientos, este estudio busca determinar de manera específica cuál es la intensidad de la relación entre capacitación y actitud hacia los riesgos laborales en tres pymes del sector construcción del área metropolitana de Bucaramanga.

\section{Método}

\section{Tipo de estudio}

La investigación tuvo un enfoque cuantitativo no experimental de corte transversal de alcance correlacional.

\section{Participantes y datos}

La población meta seleccionada fueron los empleados de tres pymes del sector construcción del área metropolitana de Bucaramanga.

Para fines del estudio se determinó un tamaño de muestra de ciento trece trabajadores, considerando un nivel de significancia del $90 \%$ y un error de estimación del $5 \%$. Los trabajadores se seleccionaron aleatoriamente en proporción al número de empleados de cada empresa. Los encuestados firmaron un consentimiento informado autorizando la utilización de la información recolectada para fines de la investigación.

\section{Materiales e instrumentos}

Con este estudio se buscaba determinar si la actitud hacia los riesgos laborales, entendida como la disposición favorable hacia la prevención de los riesgos laborales, era dependiente de las variables: i) número de capacitaciones realizadas en temas clave de prevención de riesgos laborales en el último año. ii) nivel del cargo. iii) conocimiento del plan de capacitación de la empresa. iv) percepción sobre la importancia de las capacitaciones en temas clave de prevención de riesgos laborales. v) conocimiento de las normas de seguridad. y vi) ocurrencia de accidentes laborales.

Tomando como base el instrumento creado por Estepa, Vanegas y Monsalve (2012) se diseñó la escala de actitud hacia los riesgos laborales en el sector construcción. Esta escala tiene veinte ítems redactados en sentido positivo y evaluables con una escala de Likert de cinco niveles así: $1=$ Nunca, $2=$ Casi nunca, $3=$ Algunas veces, $4=$ Casi siempre, $5=$ Siempre.

La escala se complementó incluyendo preguntas sobre la identificación de la empresa y del encuestado, el conocimiento e importancia del plan de capacitación de la empresa, los temas de las últimas capacitaciones recibidas, la ocurrencia de accidentes laborales, la percepción sobre las principales causas que favorecen la ocurrencia de accidentes de trabajo, el conocimiento de las normas de seguridad y la percepción sobre los riesgos más críticos del sector (Anexo $\mathrm{A}$ ).

Como medio de validación de la escala se utilizó el análisis factorial, mediante el cual se constató la unidimensionalidad de la escala y su utilidad para medir el constructo actitud hacia los riesgos laborales (Roche, 2010).

\section{Procedimiento}

Se entrevistaron un total de ciento trece trabajadores de las tres empresas, de los cuales treinta y tres eran directivos y mandos medios y ochenta operativos (Tabla 1). 
Tabla 1

Número de empleados encuestados por empresa y por nivel del cargo

\begin{tabular}{cccc}
\hline Empresa & $\begin{array}{c}\text { Directivos } \mathbf{y} \\
\text { mandos medios }\end{array}$ & Operativos & Total \\
\hline Empresa 1 & 19 & 31 & 50 \\
Empresa 2 & 4 & 42 & 46 \\
Empresa 3 & 10 & 7 & 17 \\
Total & $\mathbf{3 3}$ & $\mathbf{8 0}$ & $\mathbf{1 1 3}$ \\
\hline
\end{tabular}

\section{Fuente: Elaboración propia}

\section{Resultados}

El nivel de actitud hacia los riesgos laborales en el sector construcción es de 3,68 sobre 5 con una desviación estándar de 0,51, de manera que la disposición favorable hacia la prevención de los riesgos laborales de los trabajadores del sector construcción está en un nivel medio. Sin embargo, el nivel de actitud hacia los riesgos laborales varía significativamente entre empresas y niveles de cargo (ver Figura 2).

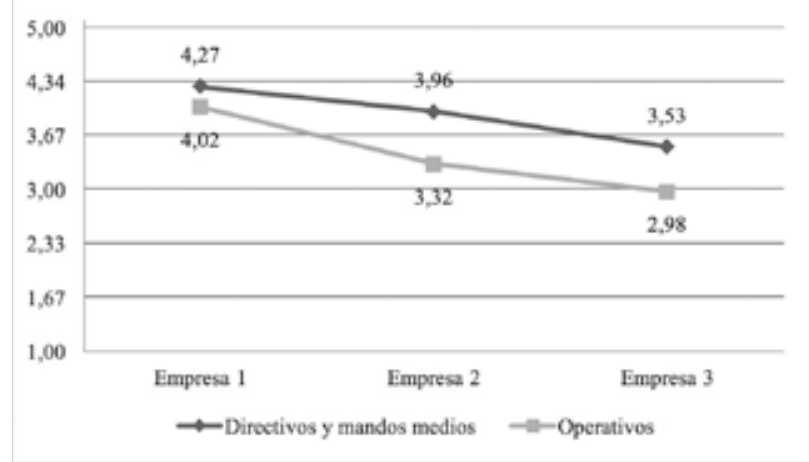

Figura 2.

Actitud promedio hacia los riesgos laborales por empresa y por nivel de cargo.

Fuente: Elaboración propia.

La prueba de normalidad de Shapiro-Wilk llevó a descartar el comportamiento normal de la variable actitud hacia los riesgos laborales para las distintas submuestras (valores P por debajo del $5 \%$ ), lo cual hizo necesario recurrir al uso de pruebas no paramétricas para determinar diferencias significativas en el nivel de actitud hacia los riesgos laborales de las empresas en los distintos niveles de cargo.

Se utilizó la prueba de Mann-Whitney para determinar si había diferencia entre la actitud hacia los riesgos laborales de los directivos y mandos medios en comparación con los cargos operativos. Como el Valor $\mathrm{P}=1,047 \times 10^{-5}$ resultó menor que $5 \%$, se descartó la hipótesis nula de no diferencia entre los grupos a favor de concluir que la actitud hacia los riesgos laborales en el grupo de directivos y mandos medios era significativamente mayor que en el grupo de cargos operativos.

Se utilizó la prueba de Kruskal-Wallis para determinar si había diferencia entre empresas con respecto a la actitud hacia los riesgos laborales de los trabajadores (Tabla 2). Esta prueba permitió concluir que la actitud hacia los riesgos laborales de los trabajadores de la empresa 1, con el mayor rango promedio, era significativamente mayor con respecto a las empresas 2 y 3 (Valor $P=7,47 \times 10^{-15}$ ).

Tabla 2

Prueba de Kruskal-Wallis para determinar diferencias entre empresas

\begin{tabular}{ccc}
\hline Empresa & $\mathbf{N}$ & $\begin{array}{c}\text { Rango } \\
\text { promedio }\end{array}$ \\
\hline Empresa 1 & 50 & 84,83 \\
Empresa 2 & 46 & 35,96 \\
Empresa 3 & 17 & 32,09 \\
Total & 113 & \\
& Chi-cuadrado & 65,057 \\
& Grados de libertad & 2 \\
& Sig. Asintótica & $\mathbf{7 , 4 6 6 \mathbf { 4 0 }} \mathbf{1 0}^{-15}$ \\
\hline
\end{tabular}

\section{Fuente: Elaboración propia}

Fue necesario aplicar la prueba de Mann-Whitney para determinar si había diferencia entre la actitud hacia los riesgos laborales de los trabajadores de las empresas 2 y 3. Como el Valor $P=0,3172$ resultó mayor que el nivel de significancia del $5 \%$, no fue posible descartar la hipótesis nula de que no había diferencia en la actitud hacia los riesgos entre los trabajadores de la empresa 2 y de la empresa 3.

Con respecto a las capacitaciones realizadas en el último año la empresa 1 realizó veinte capacitaciones de veinte programadas, la empresa 2 realizó nueve capacitaciones de quince programadas y la empresa 3 realizó cuatro capacitaciones de ocho programadas.

Del $100 \%$ de los trabajadores encuestados, el 39,82 \% dijo conocer el plan de capacitación de la empresa en temas clave de prevención de riesgos laborales, el 98,23\% consideró importante la realización de capacitaciones en temas clave de prevención de riesgos laborales, el 
$99,12 \%$ manifestó conocer las normas de seguridad para realizar trabajos con equipos y herramientas usadas en el sector construcción y el 4,42\% afirmó haber tenido accidentes laborales en los últimos dos años.

Se procedió a realizar la prueba de correlación para determinar la relación entre la actitud hacia los riegos laborales y las otras variables. La prueba de correlación mostró que la actitud hacia los riesgos laborales tiene una relación significativa con el nivel del cargo, el número de capacitaciones realizadas y el conocimiento del plan de capacitación (Tabla 3). La prueba de correlación también permitió determinar la relación entre el conocimiento del plan de capacitación y las variables número de capacitaciones realizadas y nivel de cargo.

Tabla 3

Matriz de correlación distintas variables de estudio

\begin{tabular}{|c|c|c|c|c|c|c|c|c|}
\hline & & $\begin{array}{c}\text { Actitud } \\
\text { hacia los } \\
\text { riesgos } \\
\text { laborales }\end{array}$ & $\begin{array}{l}\text { Nivel del } \\
\text { cargo }\end{array}$ & $\begin{array}{l}\text { Número de } \\
\text { capacitaciones } \\
\text { realizadas }\end{array}$ & $\begin{array}{c}\text { Importancia } \\
\text { de las } \\
\text { capacitaciones }\end{array}$ & $\begin{array}{l}\text { Conocimiento } \\
\text { del plan de } \\
\text { capacitación }\end{array}$ & $\begin{array}{l}\text { Conocimiento } \\
\text { de las normas } \\
\text { de seguridad }\end{array}$ & $\begin{array}{c}\text { Ocurrencia } \\
\text { accidentes } \\
\text { laborales }\end{array}$ \\
\hline $\begin{array}{l}\text { Actitud hacia } \\
\text { los riesgos } \\
\text { laborales }\end{array}$ & $\begin{array}{l}\text { Correlación de } \\
\quad \text { Pearson } \\
\text { Sig. (bilateral) }\end{array}$ & 1 & & & & & & \\
\hline Nivel del cargo & $\begin{array}{l}\text { Correlación de } \\
\text { Pearson } \\
\text { Sig. (bilateral) }\end{array}$ & $\begin{array}{l}0,406^{* *} \\
0,000\end{array}$ & 1 & & & & & \\
\hline $\begin{array}{l}\text { Número de } \\
\text { capacitaciones } \\
\text { realizadas }\end{array}$ & $\begin{array}{l}\text { Correlación de } \\
\quad \text { Pearson } \\
\text { Sig. (bilateral) }\end{array}$ & $\begin{array}{l}0,738^{* *} \\
0,000\end{array}$ & $\begin{array}{l}0,071 \\
0,454\end{array}$ & 1 & & & & \\
\hline $\begin{array}{l}\text { Importancia } \\
\text { de las } \\
\text { capacitaciones }\end{array}$ & $\begin{array}{l}\text { Correlación de } \\
\text { Pearson } \\
\text { Sig. (bilateral) }\end{array}$ & $\begin{array}{l}0,057 \\
0,548\end{array}$ & $\begin{array}{l}0,086 \\
0,364\end{array}$ & $\begin{array}{l}0,024 \\
0,804\end{array}$ & 1 & & & \\
\hline $\begin{array}{l}\text { Conocimiento } \\
\text { del plan de } \\
\text { capacitación }\end{array}$ & $\begin{array}{l}\text { Correlación de } \\
\quad \text { Pearson } \\
\text { Sig. (bilateral) }\end{array}$ & $\begin{array}{l}0,590^{* *} \\
0,000\end{array}$ & $\begin{array}{l}0,630^{* *} \\
0,000\end{array}$ & $\begin{array}{l}0,270^{* *} \\
0,004\end{array}$ & $\begin{array}{l}0,109 \\
0,250\end{array}$ & 1 & & \\
\hline $\begin{array}{l}\text { Conocimiento } \\
\text { de las normas } \\
\text { de seguridad }\end{array}$ & $\begin{array}{l}\text { Correlación de } \\
\quad \text { Pearson } \\
\text { Sig. (bilateral) }\end{array}$ & $\begin{array}{l}0,158 \\
0,096\end{array}$ & $\begin{array}{l}0,061 \\
0,523\end{array}$ & $\begin{array}{l}0,136 \\
0,152\end{array}$ & $\begin{array}{r}-0,013 \\
0,894\end{array}$ & $\begin{array}{l}0,077 \\
0,418\end{array}$ & 1 & \\
\hline $\begin{array}{c}\text { Ocurrencia } \\
\text { accidentes } \\
\text { laborales }\end{array}$ & $\begin{array}{l}\text { Correlación de } \\
\quad \text { Pearson } \\
\text { Sig. (bilateral) }\end{array}$ & $-0,183$ & 0,051 & $-0,099$ & 0,029 & 0,001 & 0,020 & 1 \\
\hline
\end{tabular}

**La correlación es significativa al nivel 0,01 (bilateral) Fuente: Elaboración propia

Para confirmar estos resultados se procedió a utilizar la regresión logística binaria, siguiendo el método Wald hacia atrás (Tabla 4). En primer lugar se dicotomizó la variable actitud hacia los riesgos laborales, estableciendo nivel alto $=1$ para valores de actitud mayores o iguales a 3,67 y nivel medio o bajo $=0$ para valores inferiores a 3,67 .

A continuación, se verificó el cumplimiento de los supuestos para la aplicación del modelo Independencia de errores y de no multicolinealidad de las variables independientes. El test de Durbin-Watson arrojó un valor de 2,043 , indicando el cumplimento del supuesto de independencia de errores y el Factor de Inflación de la Varianza - FIV resultó estar entre 1,014 y 8,175 para todas las variables, cumpliendo el supuesto de no multicolinealidad de las variables independientes.

El modelo tuvo un resultado final de siete pasos. El modelo se aceptó porque resultó significativo al explicar el $80,6 \%$ de la varianza de la variable dependiente y predecir correctamente el valor de la variable dependiente el $91,2 \%$ de las veces. 
Tabla 1

Equipos que componen la arquitectura de comunicaciones de subestaciones MT

\begin{tabular}{|c|c|c|c|c|c|c|}
\hline & B & Wald & Sig. & Exp (B) & $\operatorname{Exp}(B)$ & $\operatorname{Exp}(B)$ \\
\hline Constante & $-6,709$ & 31,910 & 0,000 & 0,001 & - & - \\
\hline Nivel & - & - & - & - & - & - \\
\hline Número de capacitaciones realizadas & 0,515 & 24,942 & 0,000 & 1,673 & 1,367 & 2,047 \\
\hline Importancia de las capacitaciones & - & - & - & - & - & - \\
\hline Conocimiento del plan de capacitación & - & - & - & - & - & - \\
\hline Conocimiento de las normas de seguridad & - & - & - & - & - & - \\
\hline Ocurrencia accidentes laborales & - & - & - & - & - & - \\
\hline $\begin{array}{l}\text { Conocimiento del plan de capacitación * } \\
\text { Número de capacitaciones realizadas }\end{array}$ & - & - & - & - & - & - \\
\hline Conocimiento del plan de capacitación * Nivel & 4,750 & 21,130 & 0,000 & 115,625 & 15,255 & 876,384 \\
\hline-2 log de la verosimilituda & & & & 51,534 & & \\
\hline R cuadrado de Cox y Snell & & & & 0,603 & & \\
\hline R cuadrado de Nagelkerke & & & & 0,806 & & \\
\hline Chi-cuadrado & & & & 104,400 & & \\
\hline Significación & & & & 0,000 & & \\
\hline$N$ & & & & 133 & & \\
\hline \% Global Tabla Clasificación ${ }^{b}$ & & & & $91,2 \%$ & & \\
\hline
\end{tabular}

${ }^{a}$ La estimación ha finalizado en el número de iteración 7 porque las estimaciones de los parámetros han cambiado en menos de 0,001.

${ }^{b}$ Valor de corte 0,5

Fuente: Elaboración propia

Las variables que resultaron significativas de acuerdo con el modelo fueron el número de capacitaciones realizadas y el nivel del cargo, de manera conjunta con el conocimiento del plan de capacitación. De acuerdo con esto, la probabilidad de que la actitud hacia la prevención de los riesgos laborales sea alta $(y=1)$ estaría dada por:

$$
P_{(y=1)}=\frac{1}{1+e^{-\left(-6,70920+0,51452 X_{1}+4,75035 \times X_{2} \times X_{3}\right)}}
$$

Dónde:

$\mathrm{X}_{1}=$ Número de capacitaciones

$X_{2}=\left\{\begin{array}{l}0 \text { si el trabajador es de nivel operativo } \\ 1 \text { si el trabajador es de nivel directivo o mandos } \\ \text { medios }\end{array}\right.$

$X_{3}=\left\{\begin{array}{l}0 \text { si el trabajador no conoce el plan de } \\ \text { capacitación } \\ 1 \text { si el trabajador conoce el plan de capacitación }\end{array}\right.$

De manera que, por una parte, la probabilidad de que la actitud hacia los riesgos laborales sea alta aumenta con el número de capacitaciones, y por otra, la probabilidad de que la actitud hacia los riesgos laborales sea alta para un trabajador de nivel directivo o mando medio aumenta cuando el trabajador conoce el plan de capacitación.

\section{Análisis de fiabilidad de la escala}

Acto seguido, se pasó a evaluar la fiabilidad de la escala de actitud hacia los riesgos laborales en el sector construcción con el coeficiente alfa de Cronbach. El coeficiente alfa de Cronbach de la escala de actitud hacia los riesgos laborales en el sector construcción resultó ser 0,933 , confirmando la fiabilidad del instrumento. Por otra parte, la correlación ítem-total corregida para todos los ítems estuvo por encima del 0,35, excepto para el ítem 5; sin embargo, el cálculo del coeficiente alfa de Cronbach suponiendo la eliminación del ítem 5, arrojó una mejora de tres milésimas, lo cual no justifica su eliminación.

Como el índice KMO resultó ser 0,92, se concluyó que los datos eran idóneos para realizar un análisis factorial. El análisis factorial permitió determinar un total de tres componentes, explicando el $58,42 \%$ de la varianza (Tabla 
5); sin embargo, se confirmó la unidimensionalidad de la escala en consideración a que el componente 1 agrupaba el $90 \%$ de los ítems.

Tabla 5

Distribución de la varianza por componentes

\begin{tabular}{cccc}
\hline Componentes & $\begin{array}{c}\text { Participación } \\
\text { en la varianza } \\
\text { (\%) }\end{array}$ & $\begin{array}{c}\text { Participación } \\
\text { en la varianza } \\
\text { acumulada (\%) }\end{array}$ \\
\hline 1 & 45,655 & 45,655 & $\begin{array}{r}1,2,3,4,7,8,9,10, \\
11,12,13,14,15, \\
16,17,18,19,20\end{array}$ \\
& & & 16,17 \\
2 & 7,311 & 52,965 & 6 \\
3 & 5,454 & 58,419 & 5 \\
\hline
\end{tabular}

\section{Fuente: Elaboración propia}

Se concluyó entonces que la escala de actitud hacia los riesgos laborales en el sector construcción era consistente y unidimensional, no siendo necesario realizarle modificaciones.

\section{Capacitaciones realizadas y percepción sobre los riesgos críticos}

En cuanto a las temáticas de las capacitaciones recibidas recientemente, del $100 \%$ los trabajadores encuestados, el $55,51 \%$ fue capacitado en estrés laboral, el $51,45 \%$ en riesgo ergonómico, el 40,56\% en manejo de residuos, el $30,54 \%$ en riesgos que derivan en accidentes con lesiones en las manos, $25,77 \%$ en acoso laboral y $23,16 \%$ en trabajo en alturas.

En cuanto a las principales causas de ocurrencia de accidentes de trabajo, del $100 \%$ de los trabajadores encuestados, el $100 \%$ identificó el arnés en mal estado; el $99,1 \%$, el levantamiento de cargas pesadas; el $84,1 \%$, la distracción; el 80,5\%, el exceso de confianza; el 69,9\%, el orden y limpieza deficientes, y el 44,2\% identificó las puestas a tierra inexistentes.

En cuanto a los riesgos laborales más críticos, del $100 \%$ de los trabajadores encuestados el 97,35\% identificó la manipulación de cargas; el $96,46 \%$, la caída de objetos; el $94,69 \%$ el trabajo en alturas; el $81,42 \%$, el riesgo eléctrico; el $77,88 \%$, el material particulado, y el $59,29 \%$, las vibraciones.

\section{Discusión}

La actitud hacia los riesgos laborales de los empleados encuestados resultó estar en un nivel medio-alto. El análisis por nivel de cargo mostró que los directivos y mandos medios tienen un nivel medio-alto de actitud hacia los riesgos laborales mientras que los trabajadores operativos tienen un nivel medio. Este es un aspecto clave, porque los directivos $-\mathrm{y}$ en especial los mandos medios- tienen amplia influencia en la consolidación de la cultura de seguridad (Kouabenan, Ngueutsa, \& Mbaye, 2015) que favorece la implementación del sistema de gestión de la salud y la seguridad en una organización (Health and Safety Commission, 1993)

Por otra parte, el modelo de regresión logística confirmó una relación positiva significativa entre el nivel de actitud hacia los riesgos laborales y el número de capacitaciones realizadas en el último año en temas clave de prevención de riesgos laborales: el nivel de actitud hacia los riesgos laborales de la empresa 1 es mayor que para las empresas 2 y 3 , y esto coincide con el mayor número de capacitaciones realizadas en la empresa 1. Dicho hallazgo coincide también con lo propuesto por Christian, Bradley, Wallace, \& Burke (2009) con respecto al efecto positivo que tiene el conocimiento de los riesgos con los comportamientos seguros.

También se logró determinar, mediante el modelo de regresión logística, una relación positiva significativa entre el nivel de actitud hacia los riesgos laborales y la interacción de las variables nivel del cargo y conocimiento del plan de capacitación. Esta relación tiene mayor fuerza que la relación de la actitud con respecto al número de capacitaciones; a manera de ejemplo, si la empresa realizó en el último año doce capacitaciones, la probabilidad de que un trabajador de nivel directivo que conoce el plan de capacitación tenga una actitud alta es $98,54 \%$, mientras que la probabilidad de que un trabajador de nivel directivo que desconoce el plan de capacitación tengo una actitud alta o la probabilidad de que un trabajador de nivel operativo que conoce o no el plan de capacitación tenga una actitud alta es 36,94\%.

El modelo de regresión logística, además de excluir el nivel de cargo como explicativo del nivel de actitud hacia los riesgos laborales, también llevó a excluir las variables: i) percepción sobre la importancia de las capacitaciones; ii) conocimiento del plan de capacitación; iii) conocimiento de las normas de seguridad aplicables al sector; iv) ocurrencia de accidentes laborales, y v) conocimiento del plan de capacitación * número de capacitaciones realizadas. Esto coincide con lo propuesto por Beus, Payne, Bergman \& Arthur (2010) con respecto a que los hábitos de seguridad no son predictores de accidentes laborales. 
Un hallazgo adicional del estudio es la relación entre el conocimiento del plan de capacitación y las variables número de capacitaciones realizadas y nivel de cargo. Por una parte, los directivos y mandos medios tienen mayor conocimiento del plan de capacitación de la empresa y por otra, el plan de capacitación es más conocido en las empresas con mayor número de capacitaciones realizadas.

El análisis de fiabilidad de la escala de actitud hacia los riesgos laborales en el sector construcción mediante el cálculo del alfa de Cronbach, permitió determinar su consistencia interna, no siendo necesario eliminar ítems o reagruparlos.

Este estudio permite plantear la utilización de la escala de actitud hacia los riesgos laborales en el sector construcción como un instrumento válido, tanto para medir el nivel de actitud hacia los riesgos de los trabajadores de una empresa en un momento dado, así como para medir el efecto de las capacitaciones realizadas en la actitud hacia los riesgos laborales en los trabajadores de una empresa. La sistematización de los resultados obtenidos de la aplicación de la escala en otras empresas permitirá validar sus características de fiabilidad.

Entre las limitaciones de este estudio se encuentran el impacto que puedan tener los aspectos operativos de la ejecución de la formación tales como la intensidad horaria, periodicidad, metodologías, ambientes de aprendizaje, etc. Sin embargo, un estudio adelantado por Burke et al. (2006) reportó mayor impacto de las capacitaciones presenciales con respecto a las impartidas a distancia.

\section{Referencias}

Alcántara, R., Rodríguez, M.., González, C. \& Clapes, C. (2013). Percepción de riesgo en alumnos de Enfermería. Enfermería Global, 12(1), 341-351. Recuperado de http:// www.redalyc.org/articulo.oa?id $=365834848018$

Bentler, P. \& Speckart, G. (1981). Attitudes" cause" behaviors: A structural equation analysis. Journal of Personality and Social Psychology, 40(2)

Beus, J. , Payne, S., Bergman, M. \& Arthur, W. (2010). Safety climate and injuries: an examination of theoretical and empirical relationships. The Journal of Applied Psychology, 95(4), 713-27. Recuperado de http://doi.org/10.1037/ a0019164

Burgos, A. (2014). Análisis de la cultura de prevención de riesgos laborales en los distintos niveles educativos desde la perspectiva del profesorado. EDUCAR, 50, 285-
321. Recuperado de http://www.redalyc.org/articulo. oa?id=342132463007

Burke, M., Sarpy, S., Smith, K., Chan, S., Salvador, R. \& Islam, G. (2006). Relative Effectiveness of Worker Safety and Health Training Methods. American Journal of Public Health, 96(2), 315-324. Recuperado de http://doi.org/10.2105/ AJPH.2004.059840

Cámara de Comercio de Bucaramanga. (6 de mayo de 2016). Clasificación por tamaño de las empresas inscritas en Santander. Recuperado de http://www.camaradirecta. com/temas/indicadoresantander/indicadores/ insactytam2016.htm

Corrales, C., Sánchez, C. \& Toledo, G. (2014). Estudio de la Siniestralidad en Seguridad en Empresas del Sector Construcción a partir de la Nueva Legislación Peruana. In 12th Latin American and Caribbean Conference for Engineering and Technology 1-11. Guayaquil.. Recuperado de http://www.laccei.org/LACCEI2014Guayaquil/RefereedPapers/RP262.pdf

Cortijo, J., Gómez, M. \& Samalvides, F. (2010). Cambios en conocimientos, actitudes y aptitudes sobre bioseguridad en estudiantes de los últimos años de Medicina. Revista Médica Herediana, 21(1), 27-31. Recuperado de http://www.scielo.org.pe/scielo.php?script=sci_ arttext\&pid=S1018-130X2010000100005\&lng=es\&n $r m=i s o \& t$ Ing=esChen, D. \& Tian, H. (2012). Behavior based safety for accidents prevention and positive study in China construction project. Procedia Engineering, 43, 528-534. Recuperado de http://doi.org/10.1016/j. proeng.2012.08.092

Christian, M., Bradley, J., Wallace, J. \& Burke, M. (2009). Workplace safety: a meta-analysis of the roles of person and situation factors. The Journal of Applied Psychology, 94(5), 1103-27. Recuperado de http://doi.org/10.1037/a0016172

De La Torre, G. \& Tarabla, H. (2015). Accidentes laborales, enfermedades profesionales y uso de elementos de protección personal en veterinarios de pequeños animales. InVet, 17(1), 223-227. Recuperado de http://www.redalyc. org/articulo.oa?id=179144264006

Du, X. \& Sun, W. (2012). Research on the relationship between safety leadership and safety climate in coalmines. Procedia Engineering, 45, 214-219. Recuperado de http://doi. org/10.1016/j.proeng.2012.08.146

Estepa, C., Vanegas, M. \& Monsalve, I.(2012). Conocimientos, actitudes y prácticas frente a los riesgos laborales en una empresa de construcción de la ciudad de Bucaramanga

Fasecolda. (21 de mayo de 2016). Sistema de Consulta de Información en Riesgos Laborales (RL Datos). Recuperado de https://sistemas.fasecolda.com/rpDatos/ 
Fernández, V., Mayayo,L., Chamarro, A. \& Virgili, C. (2015). Evaluando la salud laboral de los docentes de centros concertados: El Cuestionario de Salud Docente. Revista de Psicologia Del Trabajo $Y$ de Las Organizaciones, 31(3), 175-185. Recuperado de http://doi.org/10.1016/j. rpto.2015.07.001

Franco,J., Marín, A., Ocampo, L. , Quiroz, T.\& Díaz, P. (2007). Factores laborales y personales frente a la ocurrencia de accidentes de trabajo biológicos en el personal de enfermería de la clínica Villapilar ESE Rita Arango Álvarez del Pino Manizales (Caldas) 2005-2006. Revista Hacia La Promoción de La Salud, 12, 134-144. Recuperado de http://www.redalyc.org/articulo.oa?id=309126689011

Health and Safety Commission. (1993). ACSNI Study Group on Human Factors. 3rd Report: Organizing for Safety. Londres: HSC

Ismail, F., Ahmad, N., Janipha, A. \& Ismail, R. (2012). Assessing the Behavioural Factors' of Safety Culture for the Malaysian Construction Companies. Procedia - Social and Behavioral Sciences, 36(June 2011), 573-582. Recuperado de http:// doi.org/10.1016/j.sbspro.2012.03.063

Kouabenan, R., Ngueutsa, R. \& Mbaye, S. (2015). Safety climate, perceived risk, and involvement in safety management. Safety Science, 77, 72-79. Recuperado de http://doi. org/10.1016/j.ssci.2015.03.009

López, M., Andrade, R., Tarabla, D., Signorini, M. \& Molineri, A. (2014). Factores asociados con la presentación de accidentes laborales en veterinarios zootecnistas del departamento de Boyacá (Colombia). Salud Uninorte, 30(1), 23-33. Recuperado de http://www.redalyc.org/ articulo.oa?id=81730850004

Martínez, C. \& Montero, R. (2015). La cultura de la seguridad en una empresa constructora: evaluación e interpretación de sus resultados. Salud de Los Trabajadores, 23(2), 115126. Recuperado de http://www.redalyc.org/articulo. oa?id=375844217005

Meza, L. \& Camargo, J. (2015). Análisis de la relación entre la cultura organizacional y la planeación estratégica en el sector de la construcción en Bucaramanga y su área metropolitana. I+D Revista de Investigaciones UDI, 6(2), 100-110. Recuperado de http://www.udi. edu.co/revistainvestigaciones/index.php/ID/article/ viewFile/28/25\#page=1\&zoom=auto,-16,794

Molina, C. (2012). Factores individuales asociados con accidentes laborales en trabajadores afiliados a dos Aseguradoras de Riesgo Profesionales de la ciudad de Medellín, 2012. Revista Facultad Nacional de Salud Pública, 30, 36-38. Recuperado de http://www.redalyc.org/articulo. oa?id=12025811008

Olivos, M., Ávila, Á. \& Arana, B. (2008). Actitudes de estudiantes de enfermería mexicanos al manejar residuos peligrosos biologico infecciosos. Escola Anna Nery Revista de Enfermagem, 12(3), 479-484. Recuperado de http://doi. org/10.1590/S1414-81452008000300013

Ospina, M., Manrique, F. \& Ariza, E. (2009). Intervención Educativa sobre los Conocimientos y Prácticas Referidas a los Riesgos Laborales en Cultivadores de Papa en Boyacá, Colombia. Revista de Salud Pública, 11(2), 182-190. Recuperado de http://doi.org/10.1590/S0124-00642009000200003

Pucci, F. (2007). Accidentes de trabajo y condiciones de riesgo en la industria de la construcción uruguaya. In La gestión del riesgo y las crisis: personas, culturas organizacionales e instituciones. (pp. 187-224). Buenos Aires: Foncsi y el Ateneo, Universidad de San Andrés y. Recuperado de http://repositorio.udesa.edu.ar/jspui/ bitstream/10908/10681/10/La gesti\%25C3\%25B3n del riesgo y la crisis. J. Walter y F. Pucci - COMPLETO.pdf

Puyal-Español, E. (2001). La conducta humana frente a los riesgos laborales: determinantes individuales y grupales. Acciones e investigaciones sociales, (12), 157-184. Recuperado de https://papiro.unizar.es/ojs/index.php/ais/ article/view/199/193

Roche, M. (2010). Usos del análisis factorial para la construcción y validación de escalas. In Curso técnico de medición multidimensional de la pobreza y sus Aplicaciones. Recuperado de http://interwp.cepal.org/mmp/pres/15_ Análisis_Factorial_Escalas.pdf

Sánchez, F., Rubio, J., Páez, D. \& Blanco, A. (1998). Optimismo ilusorio y percepción de riesgo. Boletín de Psicología, 58, 7-17

Shin, M., Lee, S., Park, M., Moon, M. \& Han, S. (2014). A system dynamics approach for modeling construction workers' safety attitudes and behaviors. Accident. Analysis and Prevention, 68, 95-105. Recuperado de http://www.sciencedirect.com/science/article/pii/ S0001457513003734

Soto, M. \& Mogollón, E. (2005). Actitud hacia la prevención de accidentes laborales de los trabajadores de una empresa de construcción metalmecánica. Salud de Los Trabajadores, 13(2), 119-123. Recuperado de http://dialnet.unirioja.es/ servlet/articulo?codigo $=1393175$

Taufek, B., Zulkifle, B. \& Kadir, B. (2016). Safety and Health Practices and Injury Management in Manufacturing Industry. Procedia Economics and Finance, 35(October 2015), 705-712. Recuperado de http://doi.org/10.1016/ S2212-5671(16)00088-5

Vallerand, J. (1994). Les fondements de la psychologie sociale. Paris: Gaëta Morín

Weinstein, D. (1989). Effects of personal experience on selfprotective behavior. Psychological Bulletin, 105(1), 
31-50. Recuperado de http://doi.org/http://dx.doi. org/10.1037/0033-2909.105.1.31

Wilde, J.(1982). The Theory of Risk Homeostasis: Implications for Safety and Health. Risk Analysis, 2(4). Recuperado de http://doi.org/10.1111/j.1539-6924.1982.tb01384.x

Zahoor, H., Chan, P., Utama, P. \& Gao, R. (2015). A Research Framework for Investigating the Relationship between Safety Climate and Safety Performance in the Construction of Multi-storey Buildings in Pakistan. Procedia Engineering, 118, 581-589. Recuperado de http://doi.org/10.1016/j. proeng.2015.08.488
Zamorano, B., Parra,V., Peña, F. \& Castillo, Y. (2009). Conocimiento y actitud en prevención de trabajadores lesionados de una empresa metalmecánica en México. Salud de Los Trabajadores, 17(1), 49-57. Recuperado de http://www. scielo.org.ve/pdf/st/v17n1/art05.pdf

Zin, M. \& Ismail, F. (2012). Employers' Behavioural Safety Compliance Factors toward Occupational, Safety and Health Improvement in the Construction Industry. Procedia - Social and Behavioral Sciences, 36(June 2011), 742-751. Recuperado de sbspro.2012.03.081 http://doi.org/10.1016/j. 


\section{Anexo A.}

\section{Cuestionario para medir actitud hacia los riesgos laborales en el sector construcción}

Este cuestionario busca medir la actitud hacia los riesgos laborales de los trabajadores del sector construcción de Bucaramanga. La información suministrada será tratada con absoluta confidencialidad y sólo será utilizada con fines académicos. De antemano gracias por su tiempo.

\begin{tabular}{|l|l|l|l|l|}
\hline \multicolumn{2}{|l|}{ Nombre de la empresa: } & \multicolumn{2}{l|}{ Sector: } & \multicolumn{2}{l|}{ No. de empleados: } & $\begin{array}{l}\text { Encuesta } \\
\text { No.: }\end{array}$ \\
\hline Dirección: & & Teléfono: & NIT: \\
\hline Nombre del encuestado: & Cargo del encuestado: & Encuestador \\
\hline
\end{tabular}

Valora con 1, 2, 3, 4, 5 cada uno de los enunciados siguientes de acuerdo con la siguiente escala: $1=$ Nunca, 2= Casi nunca, $\quad 3$ =Algunas Veces, 4 = Casi siempre, 5 = Siempre. Haz un círculo donde corresponda.

1. Si observa un compañero trabajando en alturas sin el debido equipo de protección, usted sigue su trabajo normalmente asumiendo que él es idóneo en lo que hace.

2. Su supervisor le da la orden de cargar dos bultos de cemento de $50 \mathrm{~kg}$, usted los levanta inmediatamente sin tener en cuenta la normatividad vigente para el manejo de cargas por persona.

3. Usted muestra interés cuando es citado a asistir a un entrenamiento práctico sobre trabajo seguro en alturas.

4. Cuando Usted es citado a una capacitación sobre liderazgo y crecimiento personal asiste con entusiasmo y disposición.

5. Pensar en su familia le motiva a usted a trabajar de forma segura.

6. A Usted le parece necesario contar con elementos de protección personal para realizar trabajo en alturas.

7. Cuando recibe capacitación sobre los riesgos a los que está expuesto, considera que es algo importante para la labor que realiza.

8. Cuando es citado a una capacitación sobre Normas y recomendaciones de Seguridad y salud en su trabajo usted está dispuesto a asistir.

9. Si su jefe le solicita cargar unas cajas de cerámica que pesan $60 \mathrm{~kg}$, Usted solicita ayuda de otro compañero ya que esta carga podría lesionarlo.

10. Si observa un compañero trabajando con electricidad sin la debida protección, Usted lo reporta con el jefe inmediato para que tome las medidas necesarias.

11. Utiliza los elementos de protección personal dispuestos en los programas de capacitación impartidos en la inducción de su trabajo.

12. Si le solicitan realizar con urgencia un trabajo en la obra a más de $1.50 \mathrm{~m}$, Usted se toma su tiempo para realizar la acción usando las protecciones necesarias sin importar que esto retrase la actividad.

13. Asiste a las capacitaciones programas por la empresa, aun cuando empresa se encuentre sindicalizada y le ordenen no asistir.

14. Si la empresa tiene un espacio para las pausas activas, usted normalmente cumple con esos tiempos para realizar esas tareas.

15. Sugiere capacitaciones a sus superiores de acuerdo a situaciones peligrosas que va observando en el día a día de su labor.

16. Si está utilizando una herramienta eléctrica y esta presenta fallas Usted detiene su labor y reporta la herramienta a su jefe inmediato.

17. Usa usted herramientas mecánicas que le ayuden a evitar el sobresfuerzo en el levantamiento de cargas pesadas.

\begin{tabular}{|c|c|c|c|c|}
\hline 1 & 2 & 3 & 4 & 5 \\
\hline 1 & 2 & 3 & 4 & 5 \\
\hline 1 & 2 & 3 & 4 & 5 \\
\hline 1 & 2 & 3 & 4 & 5 \\
\hline 1 & 2 & 3 & 4 & 5 \\
\hline 1 & 2 & 3 & 4 & 5 \\
\hline 1 & 2 & 3 & 4 & 5 \\
\hline 1 & 2 & 3 & 4 & 5 \\
\hline 1 & 2 & 3 & 4 & 5 \\
\hline 1 & 2 & 3 & 4 & 5 \\
\hline 1 & 2 & 3 & 4 & 5 \\
\hline 1 & 2 & 3 & 4 & 5 \\
\hline 1 & 2 & 3 & 4 & 5 \\
\hline 1 & 2 & 3 & 4 & 5 \\
\hline 1 & 2 & 3 & 4 & 5 \\
\hline 1 & 2 & 3 & 4 & 5 \\
\hline 1 & 2 & 3 & 4 & 5 \\
\hline
\end{tabular}


18. Cuando labora en superficies deslizantes se asegura de contar con un calzado adecuado para realizar esa actividad.

19. Usted acostumbra leer el manual de instrucciones de la maquinaria antes de usarla.

20. Realiza las pausas activas muy puntualmente cuando realiza labores repetitivas.

21. ¿Conoce el plan de capacitación de la empresa con respecto a los riesgos laborales a los que está expuesto en su labor diaria? 1. Si 2. No

22. ¿Considera importante para su desarrollo laboral las capacitaciones recibidas y percibe que le ayudaran a reconocer los riesgos a los que está expuesto? 1 . Si 2. No

23. Por favor señale los tres temas en los que requiere ser capacitado con prioridad:

\begin{tabular}{|l|l|l|}
\hline a. Trabajo en alturas. & g. Ahorro de agua y energía eléctrica. & \\
\hline b. Riesgo ergonómico. & & h. Acoso laboral. \\
\hline $\begin{array}{l}\text { c. Trabajo en espacios } \\
\text { confinados. }\end{array}$ & $\begin{array}{l}\text { i. Riesgos que derivan en accidentes con lesiones en las } \\
\text { manos. }\end{array}$ & \\
\hline d. Manejo de residuos. & j. Trabajo en equipo & \\
\hline e. Estrés laboral. & k. Extintores & $\begin{array}{l}\text { I. En caso de tener otra capacitación en otro aspecto } \\
\text { por favor menciónela: }\end{array}$ \\
\hline $\begin{array}{l}\text { f. Aspectos e impactos } \\
\text { ambientales. }\end{array}$ &
\end{tabular}

24. ¿Usted conoce las normas de seguridad para realizar trabajos con equipos y herramientas usadas en el sector construcción? 1. Si 2. No

25. ¿Ha tenido accidentes laborales en su actividad de construcción, en los últimos dos años? 1. Si

2. No

26. Del siguiente listado, elija las principales causas que favorecen la ocurrencia de accidentes de trabajo. Marque en todas las opciones

\begin{tabular}{|l|l|l|l|}
\hline a. La distracción & $\begin{array}{l}\text { c. Orden y limpieza } \\
\text { deficiente }\end{array}$ & $\begin{array}{l}\text { e. Arnés en mal estado } \\
\text { d. Puestas a tierra } \\
\text { inexistentes }\end{array}$ & $\begin{array}{l}\text { f. Levantamiento de } \\
\text { cargas muy pesadas. }\end{array}$ \\
\hline Otro. Cuál? & & \\
\hline
\end{tabular}

27. De la siguiente lista de riesgos, seleccione los que son críticos o de alto riesgo en su trabajo. Marque en todas las opciones

\begin{tabular}{|l|l|l|l|}
\hline $\begin{array}{l}\text { a. Riesgo Mecánico: } \\
\text { Caída de objetos }\end{array}$ & $\begin{array}{l}\text { d. Riesgo Biomecánico: } \\
\text { manipulación manual de } \\
\text { cargas }\end{array}$ & $\begin{array}{l}\text { g. Riesgo biomecánico: } \\
\text { Movimiento repetitivo } \\
\text { en alturas }\end{array}$ & $\begin{array}{l}\text { h. Riesgo físico: } \\
\text { Vibraciones }\end{array}$ \\
\hline $\begin{array}{l}\text { b. Riesgo Biológico: } \\
\text { Virus, infecciones }\end{array}$ & $\begin{array}{l}\text { f. Riesgo psicosocial: } \\
\text { Estrés }\end{array}$ & material particulado
\end{tabular}

